\title{
Control de un sistema de energía basado en supercondensadores para mitigar los huecos de tensión en el punto de conexión común
}

\author{
Carlos Orellana Uguña1* , Luis González Morales ${ }^{1}$, Filipe Soares Oliveira², Nuno Abreu Sousa²
}

'Departamento de Eléctrica, Electrónica y Telecomunicaciones, Universidad de Cuenca, Ecuador 2Departamento de Engenharia Eletroténica, Escola Superio de Tecnología e Gestãa, Instituto Politécnico de Leiria, Portugal

*Autor para correspondencia / Corresponding autor: carl.mauryou@yahoo.es

\section{Control of a system of energy based on supercapacitors to mitigate the voltage gaps at the point of common coupling}

\begin{abstract}
This article presents the design of an energy injection system based on supercapacitors in order to reduce the voltage gaps caused by the random connection of electric machines that are used in industrial activities, with the aim of improving the quality of energy at the common connection point. Therefore, a model of the energy distribution of the studied case, the energy storage that is composed of supercapacitors, the twoway energy conversion and the current and voltage control systems are made. The designed system allows the injection of a power of $22,8 \mathrm{~kW}$ with a capacity of $1.2 \mathrm{Wh}$, compensating the transients produced by the connected loads to the network in order to keep the voltage levels within the established standards given by IEEE 1159, CONELEC 004/01 y NP EN 50160-2010.
\end{abstract}

Keywords: Voltage sags, supercapacitor bank, Power conversión (AC/DC, DC/AC), point of common coupling (PCC).

\section{Resumen}

Este artículo presenta el diseño de un sistema de inyección de energía basado en supercondensadores para mitigar los huecos de tensión originados por la conexión

Editado por /

Edited by:

Lionel Trojman \& Eva

O.L. Lantsoght

Recibido /

Received:

26/09/2017

Aceptado /

Accepted:

23/01/2020

Fecha de

publicación/

Published online:

$15 / 06 / 2020$ aleatoria de máquinas eléctricas que son utilizadas en las actividades industriales, con el objetivo de mejorar la calidad de energía en el punto de conexión común. Para ello se modela el sistema de distribución de energía del caso de estudio, el sistema de almacenamiento de energía que está compuesto por supercondensadores, el sistema de conversión de energía bidireccional y el sistema de control de corriente y voltaje. El sistema diseñado permite inyectar una potencia de 22,8 kW y capacidad de 1,2 Wh, compensando los transitorios producidos por las cargas conectadas a la red para mantener los niveles de tensión dentro de las normas establecidas por IEEE 1159, CONELEC 004/01 y NP EN 50160-2010. 
Palabras clave: Huecos de tensión, Banco de supercondensadores, convertidores de potencia (AC/DC, DC/AC), punto de conexión común (PCC).

\section{INTRODUCCIÓN}

Una buena planificación del sistema de alimentación de energía involucra una rápida respuesta ante problemas que se puedan originar por motivos atmosféricos, por intervención humana o por mantenimiento; otra consideración que se debe tener presente es el continuo crecimiento de la demanda, obligando al redimensionamiento y a la expansión de la red de distribución [1] con el objetivo de incrementar los niveles de confiabilidad en el suministro y en la calidad de energía en el punto común de conexión (PCC) [2], siempre considerando los aspectos económicos para determinar si la mejor opción es el redimensionamiento, repotenciación o la interconexión de un sistema de inyección de energía en la red, cualquiera de las opciones que se implemente tendrá el objetivo de hacer cumplir las regulaciones técnicas a las empresas distribuidoras, con la finalidad de mantener la calidad de energía entregada. Para llevar a cabo este objetivo se ha implementado diferentes sistemas de almacenamiento (ESS) y estabilizadores de energía, los cuales están indicados en la Fig. 1.

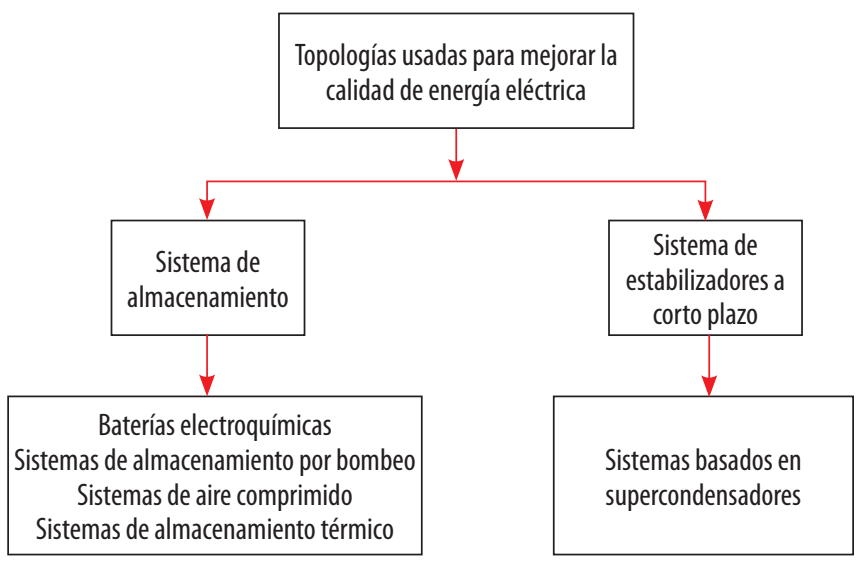

FIGURA 1. Topologías utilizadas para mejorar la calidad de energía

Estos diferentes sistemas de almacenamiento tendrán la función de absorber la energía durante períodos no picos de consumo, cuando el costo de generación es bajo o cuando la generación es excedente y abastecer la demanda durante los períodos de carga máxima cuando los costos son altos o cuando no se disponga de otro medio de generación. Sin embargo, se debe tener presente algunas consideraciones antes de su elección: tiempo de vida útil, eficiencia y ciclo de carga/descarga, las cuales están indicadas en la Fig. 2. 


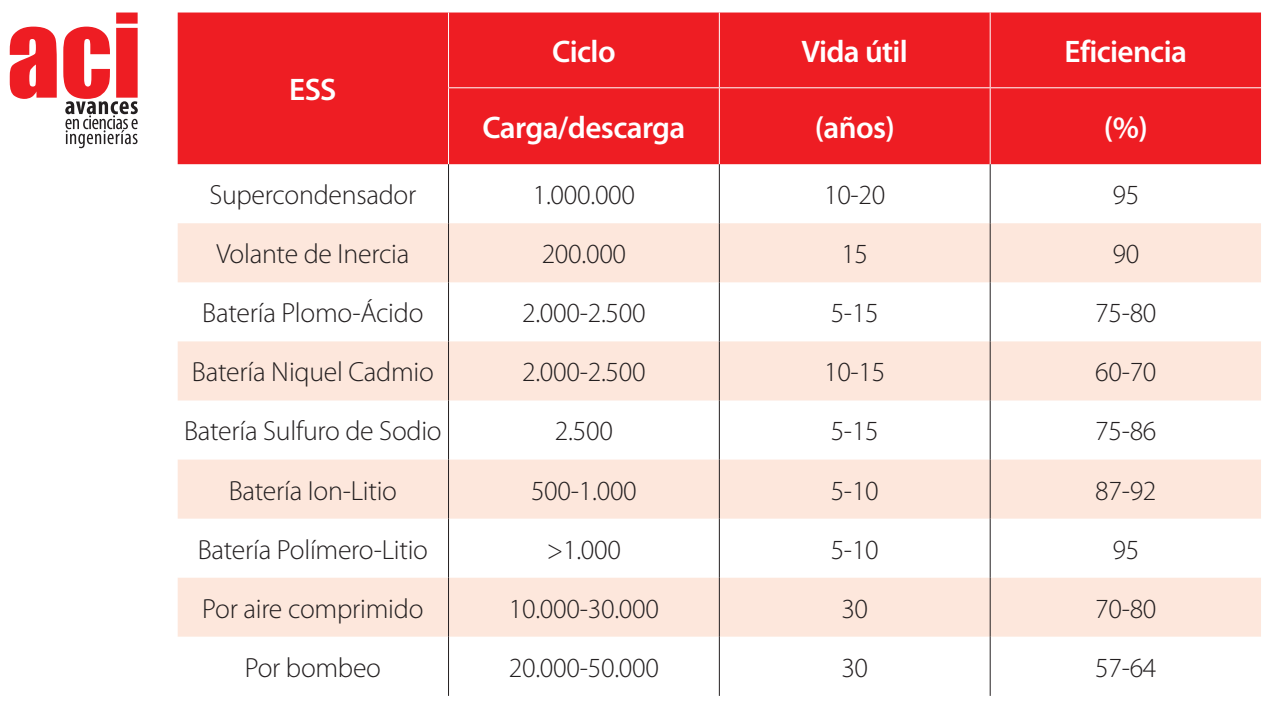

FIGURA 2. Características de los sistemas de almacenamiento de energía eléctrica

Si se considera los aspectos mencionados en la Fig. 2, la opción viable es la utilización de supercondensadores, es por ello, que el presente trabajo realiza el estudio sobre el diseño de un sistema de inyección de energía basado en supercondensadores para mitigar los huecos de tensión en el punto de conexión común.

Para verificar el correcto funcionamiento del sistema de inyección de energía se procede a modelar el sistema basado en supercondensadores con sus respectivos lazos de control y la red de distribución, mediante la utilización del simulador PSIM.

El diseño es aplicado a una zona rural con explotación minera/agrícola que presentan cargas del tipo máquinas eléctrica.

\section{DESCRIPCIÓN DEL PROBLEMA}

Debido al incremento de cargas conectadas a un PCC perteneciente a zonas con vocación minera/agrícola, la calidad del suministro de energía disminuye, dado que las características del sistema de distribución existente y a la continua puesta en marcha de las máquinas eléctricas de gran capacidad sin contar con sistemas de arranques suaves contribuyen a la formación de huecos de tensión.

Para determinar las zonas de mayor explotación minera/agrícola en el Ecuador se consideró la base de información del Instituto Nacional de Estadística y Censo (año 2010), los cuales determinaron que el 58\% de las concesiones dedicadas a la explotación minera se encuentra en ocho provincias del país: El Oro, Pichincha, Azuay, Orellana, Sucumbíos, Zamora Chinchipe, Guayas y Esmeralda, especificando lo siguiente [3]: 
- En la provincia del Oro: 6.745 personas trabajaron en la actividad de explotación minera, representado un 2,7\% del total de habitantes.

- En la provincia de Pichincha: 6.245 personas trabajaron en la actividad de canteras, representando el $0,5 \%$ del total de habitantes.

- $\quad$ En la provincia del Azuay (Camilo Ponce Enríquez, Pucará): 4.728 personas trabajan en la explotación minera, representado más del 85\% del total de habitantes, además se dedican a otras actividades como la agricultura y la ganadería [4].

A partir de lo mencionado, las zonas con mayores actividades mineras/agrícolas son: Camilo Ponce Enríquez y Pucará, por ende, en estos cantones se enfocará el estudio.

Una vez establecido las zonas de estudio, mediante el Geoportal de la Empresa Eléctricas Regional Centro Sur, perteneciente a la provincia del Azuay del país Ecuador, se procedió a identificar el alimentador que suministra de energía eléctrica, determinando que es el alimentador 1424.

Debido a la falta de existencia de normativas técnicas que regulen la potencia de los motores usados en las diferentes actividades, se consideró como referencia las maquinarias empleadas en una principal minera denomina TRES CHORRERAS [5] que establece la utilización de motores Allis Chalmers de $32 \mathrm{Hp}$, en función de ello y en base a catálogos disponibles en el Ecuador se utiliza motores eléctricos W21 a Prueba de Explosión NEMA Premium Efficiency de 50 HP que dan movimiento a bombas centrífugas para el manejo de líquidos, trituradoras, cintas transportadoras entre otras [6]. Por ende, la carga a modelar serán para esta aplicación motores eléctricos de inducción con las características indicadas en la Fig. 3 [7] y con el modelo de simulación y sus características de la red de distribución indicadas de igual forma en la Fig. 3, con resistencias de $20 \Omega$ en el modelo, la cual emula pequeños aparatos eléctricos conectados (iluminación, electrodomésticos).

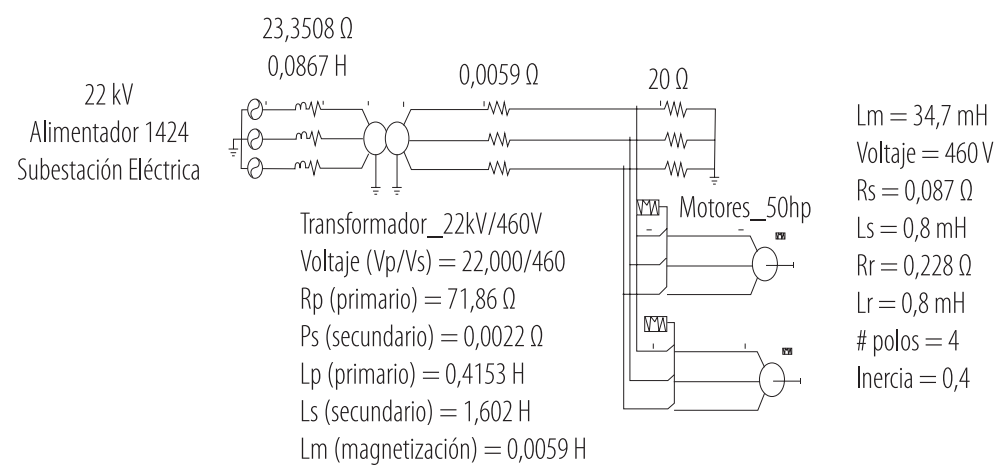

FIGURA 3. Modelo de la red de distribución

\section{CALIDAD DE ENERGÍA}

Uno de los aspectos importantes relacionadas con la calidad de energía son los llamados huecos de tensión (sag) descritos en detalle en las normativas técnicas IEEE 1159, CONELEC 
004/01 y NP EN 50160-2010, las cuales definen al sag como una disminución de la tensión de alimentación a un valor situado entre el 10\% y el 90\% de la tensión nominal, además definen a la elevación de tensión (swell) como una elevación de tensión de alimentación a un valor situado entre el $110 \%$ y $180 \%$ de la tensión nominal seguida del restablecimiento de la tensión después de un corto lapso de tiempo, aplicando este criterio al nivel de tensión fase-neutro del lado secundario del transformador de la figura 3, el rango de voltaje de fase para la formación de los sags está comprendido entre $26,55 \mathrm{~V}$ y $238,95 \mathrm{~V}$ y para el swell entre $292,05 \mathrm{~V}$ y $477,9 \mathrm{~V}$, resultando así un rango permisible de $238,95 \mathrm{~V}$ y 292,05 V en donde el nivel de tensión se considera como aceptable.

\section{PROPUESTA DEL SISTEMA DE ENERGÍA PARA MITIGARAR LOS HUECOS DE TENSIÓN EN EL PUNTO DE CONEXIÓN COMÚN}

El modelo que este estudio propone para mitigar los huecos de tensión en el PCC está indicado en la Fig. 4, el cual está compuesto por un banco de supercondensadores y un convertidor de potencia trifásico bidireccional.

Sistema de control de tensión de la ser de distribución y tensión del banco de supercondensadores

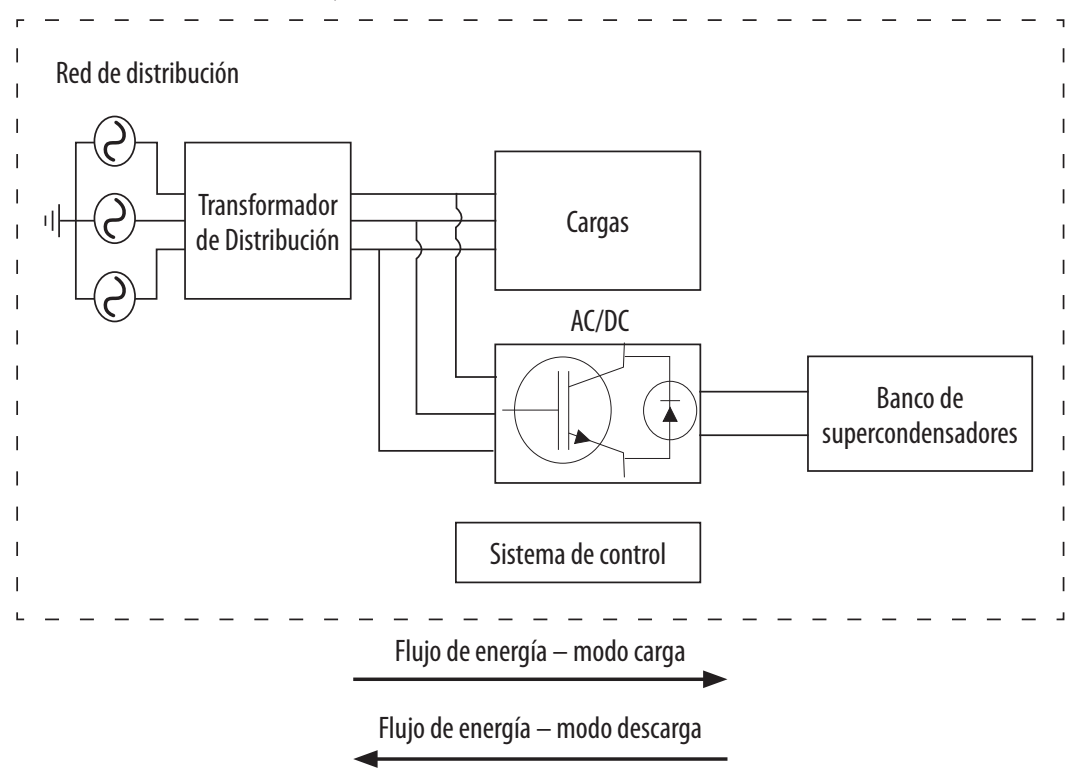

FIGURA 4. Sistema basado en supercondensadores para mitigar los huecos de tensión en el PCC

El sistema de control de la Fig. 4 consta de un control de corriente en el sistema de referencia síncrono [8,9], y tendrá la tarea de controlar la tensión de la red de distribución y tensión del banco de supercondensadores, este control se puede dividir en tres subetapas: 
- Modo carga, la energía fluye desde la red de distribución hacia el banco de supercondensadores para alcanzar el nivel de tensión nominal, en este modo, opera el sistema de control de tensión del banco de supercondensadores comportándose el convertidor como rectificador trifásico controlado.

- Modo descarga, la energía fluye desde el banco de supercondensadores hacia la red de distribución, en este modo, opera el sistema de control de tensión de la red de distribución comportándose el convertidor como inversor trifásico controlado.

- Modo stand-by, no existe circulación de energía debido a que el nivel de tensión de los supercondensadores coincide con la tensión de referencia establecido, en este modo, el convertidor trifásico se encuentra inactivo esperando el momento exacto para convertirse en inversor controlada para inyectar energía desde los supercondensadores hacia a la red eléctrica o en rectificador controlado para inyectar energía desde la red eléctrica hacia los supercondensadores.

A continuación, se describe el lazo de control que conforma el sistema basado supercondensadores.

\section{Sistema de control perteneciente al control de la tensión de la red de distribución y tensión del banco de supercondensadores}

El sistema de control, cuenta con una estructura definida por el modo de operación "carga o descarga", la Fig. 5, indica en forma de diagrama de bloques el sistema de control en cascada, donde el lazo interno de control es el correspondiente a las corrientes en el eje directo $\left(i_{d}\right)$ y cuadratura ( ).

En base al nivel de tensión que soporta el convertidor trifásico SKiM459GD12E4 y el banco de supercondensadores U37F tipo E37F501CPN103MFK0M, se establece que para el modo carga, el valor de referencia de la tensión del banco de supercondensadores se fijará en $900 \mathrm{~V}$.

Modo carga

Contro de la tensión del banco de supercondensador

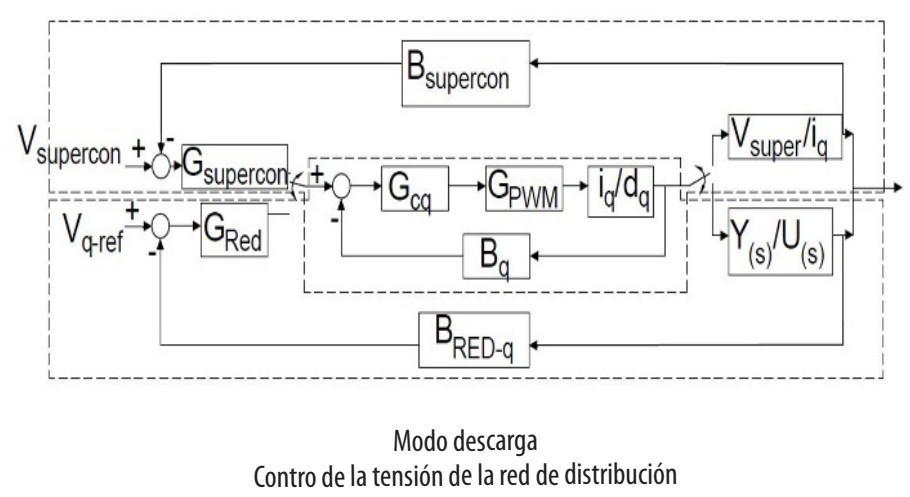

FIGURA 5. Lazos de control para la tensión de la red de distribución y del banco de supercondensadores 
De la Fig. 5, $B_{q^{\prime}} B_{\text {(Red-ay }} B_{\text {supercon }}$ son las ganancias asociadas a los censados de corriente, de tensión de la red de distribución y de la tensión del banco de supercondensadores respectivamente, con valores unitarios, $G_{c d} G_{\text {supercon' }} G_{\text {RED }}$ son las funciones de transferencia de los controladores, que en esta aplicación se han utilizado controladores clásico proporcionales e integrales PI , G ${ }_{g w m}$ es la ganancia del PWM, que en este caso se ha utilizado con ganancia unitario, $\frac{Y(S)}{U(S)}$ representa la función de transferencia que relaciona

la tensión de la red de distribución con respecto a la corriente en el eje de cuadratura, $\frac{V_{\text {super }}}{\tilde{I}_{q}}$ representa la función de transferencia que relaciona la tensión del banco de supercondensadores respecto a la corriente en el eje de cuadratura.

Las ecuaciones del balance de energía asociada al banco de supercondensadores pueden representarse mediante las ecuaciones (1) y (2) [8].

$$
\begin{aligned}
\frac{V_{\text {super }}}{\tilde{I}_{q}} & =\frac{D_{q}}{C s^{\prime}+\frac{l_{\text {filtro }}}{V_{\text {banco_super }}}} \\
D_{q} & =\frac{2 \sqrt{2 V}_{f-n}}{V_{\text {banco_super }}}
\end{aligned}
$$

Donde: $D_{q}$ es el índice de modulación, $C$ es la capacitancia total del banco de supercondensadores, $I_{\text {filtro }}$ es la corriente que circula desde el puente rectificador hacia el banco de supercondensadores, $V_{\text {banco_super }}$ ) es la tensión nominal del banco de supercondensadores, $s^{\prime}$ es el operador de Laplace.

Para calcular el valor de la capacitancia total del banco de supercondensadores se hace uso de la ecuación (3).

$$
C=\frac{Q}{V}
$$

Dónde: $Q$ es la carga en Coulomb, definido como la integral de la corriente $i(t)$ que circula a través del banco de supercondensadores, $t$ es el tiempo en el cual se produce el arranque de los motores (que para este caso es de 0,2 s) y V es la diferencia de tensión que se requiere al momento de descargarse el banco de supercondensadores que en este caso se considerará una diferencia de $30 \mathrm{~V}$.

El modelo de la Fig. 6, permite calcular el valor de $\int_{0}^{t} i(t)$.

La carga de la Fig. 6 está conformado por las características indicadas en la Fig. 3. 


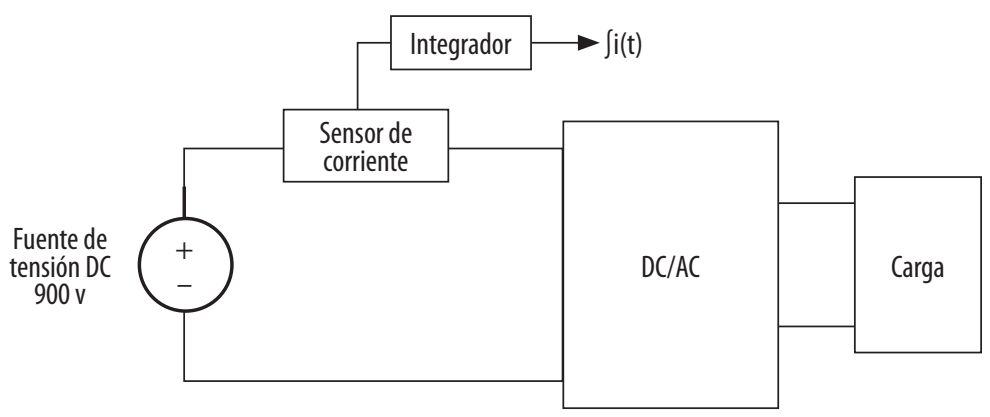

FIGURA 6. Modelo para el cálculo de la integral de la corriente

Al aplicar el modelo de la Fig. 6 en la ecuación (3) se obtuvo un valor de capacitancia total de 0,1 F, pero se debe considerar los siguientes aspectos:

- El nivel de tensión que debe soportar el banco de capacitores es de $900 \mathrm{~V}$, para ello se usó dos capacitores en serie de 0,01 F con una tensión máxima individual de soporte de $550 \mathrm{~V}$.

- La capacitancia total debe de ser de 0,1 F, como se usó dos capacitores en serie de $0,01 \mathrm{~F}$ resulta la capacitancia total de 0,005 F, por lo tanto, para que la capacitancia total sea de 0,1 F se hará uso de 20 capacitores en paralelo.

De igual modo el modelo del convertidor de potencia, puede tratarse mediante la ecuación (4) [8].

$$
\frac{\tilde{l}_{d(s)}{ }^{\prime}}{\tilde{d}_{d(s)}{ }^{\prime}}=\frac{\tilde{l}_{q(s)}{ }^{\prime}}{\tilde{d}_{q(s)}{ }^{\prime}}=\frac{V_{0}}{L s^{\prime}+R_{s}}
$$

Para los parámetros involucrados en la ecuación (4), se considera lo siguiente:

- $\quad V_{0}$ es la tensión del banco de supercondensadores, en el presente trabajo se ha definido en un valor de $900 \mathrm{~V}$.

- L L la inductancia del filtro de entrada de acuerdo a 4 [10].

- Para el cálculo se procede de la siguiente manera: se considera que la reactancia inductiva va a ser igual al $10 \%$ de la resistencia base [8] resultando y a partir de ello . A partir de este valor inicial se calibra el valor del filtro $L$ hasta llegar a un valor de $7 \mathrm{mH}$, con el cual el THD se encuentra dentro de la norma NP EN 50160-2010, CONELEC 004/01 (menor al 8\%).

- $\quad R_{s}$ está asociado a las pérdidas de los elementos pasivos y semiconductores que existe en el convertidor [10].

- $\quad s^{\prime}$ es el operador de Laplace. 
En el caso del modo descarga, el convertidor de potencia controlado mediante los lazos de corriente en el eje directo y cuadratura son utilizado para el control de la tensión en la red de distribución mediante la función de transferencia $\left(\frac{Y(s)}{U(s)}\right)$ que para efecto del diseño del sistema de control se ha caracterizado a una aproximación de un sistema de primer orden.

Para encontrar los parámetros que constituyen dicha función de trasferencia se considera que el sistema basado en banco de supercondensadores va a estar conectado en paralelo a la red de distribución comportándose como fuente de corriente, de forma gráfica, la Fig. 7, indica el esquema para determinar la función de trasferencia ante la respuesta escalón.

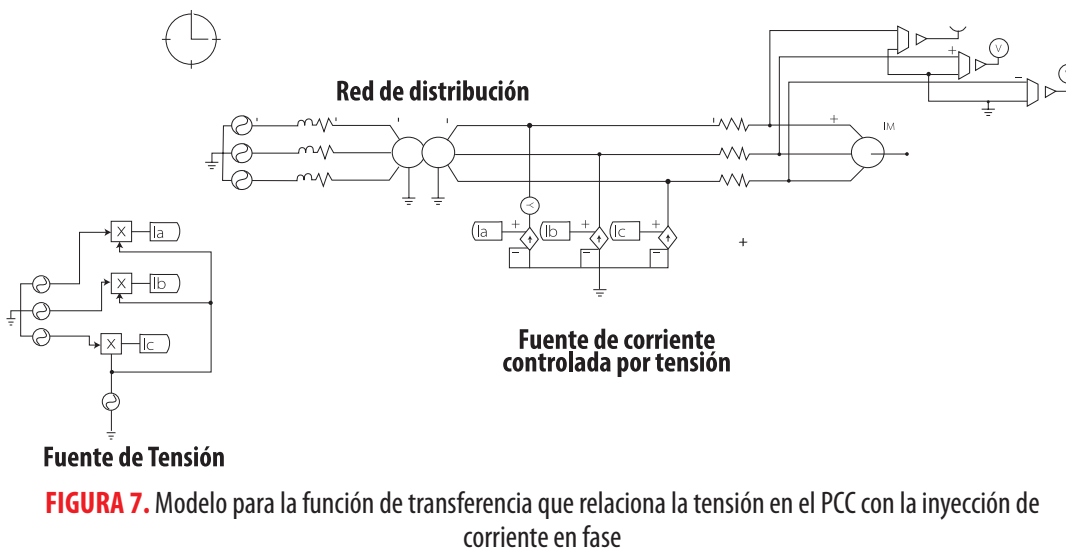

Al aplicar el procedimiento antes descrito, el comportamiento de la tensión en la red de distribución ante perturbaciones de corriente con amplitud de, se obtiene, obteniéndose una función de transferencia como la que se observa en la ecuación (5).

$$
\frac{Y_{(s)}}{U_{(s)}}=\frac{0,129}{0,287 s^{\prime}+1}
$$

Es importante destacar que las corrientes que se inyecten desde el sistema de almacenamiento de energía hacia el PCC deben constar con un sistema de sincronización de fase para poder tener un control de energía activa o reactiva, en este diseño se ha utilizado un sistema de enganche de fase PLL [8].

Un inconveniente que presenta este sistema de sincronización de fase, es que, una vez sintonizado el controlador PI para un determinado valor de amplitud de la entrada, al cambiar la misma, varía la dinámica del lazo con llevando a que de nuevo se vuelva a sintonizar el PI. Una solución para ello es la normalización de las magnitudes de entrada [8], es decir, ante cualquier amplitud de valor de entrada con un bloque de trasformación, esta magnitud siempre será igual a uno. Para realizar esta normalización se utiliza la ecuación (6) con un modelo igual al de la Fig. 8 [8]. 


$$
\left[\begin{array}{l}
x_{1} \\
x_{2}
\end{array}\right]=\left[\frac{\frac{e_{\alpha}}{\sqrt{e_{\alpha}{ }^{2}+e_{\beta}^{2}}}}{\frac{e_{\beta}}{\sqrt{e_{\alpha}{ }^{2}+e_{\beta}^{2}}}}\right]
$$

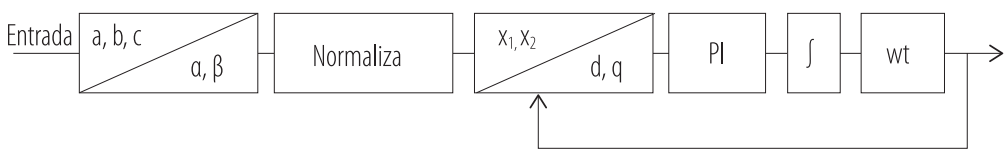

FIGURA 8. Diagrama de un PLL normalizado

La sintonización de los PI para los diferentes lazos de control se realiza por asignación de polos y ceros tomando en consideración los siguientes aspectos [8]:

- La frecuencia de conmutación en este trabajo se ha fijado en 15 kHz, lo que limita las dinámicas de los lazos de corriente, dado que para frecuencias superiores a $\frac{f_{s w}}{2}=$ $7,5 \mathrm{kHz}$, la técnica del modelado pierde validez.

- A parir de la frecuencia de sintonización del PI para el lazo de corriente, se debe sintonizar una década menos para los lazos restantes de control.

- El margen de fase del diagrama de Bode tiene que ser mayor o igual a $60^{\circ}$.

- $\quad$ El margen de ganancia mayor o igual a 7dB.

Considerando estos aspectos se obtuvieron los siguientes parámetros del PI:

- $\quad$ Sistema de control: para el lazo de corrientes en el eje directo y cuadratura el controlador PI posee un $k_{p}=0,36$ y $, k_{i}=0,09$ para el lazo de control de tensión del banco de supercondensadores el controlador PI posee un $k_{p}=15$ y $k_{i}=0,03$ y para el lazo de control de tensión de la red de distribución el controlador PI posee un $k_{p}=16,47$ y $k_{i}=0,027$.

- $\quad$ Para el sistema de sincronización de fase PLL, el controlador PI posee un $k_{p}=44.312,4$ y $k_{i}=1.007,1$.

El sistema de inyección de energía basado en banco de supercondensadores en modo descarga posee una restricción, la cual tiene que ver con la mínima tensión que puede llegar en el momento de inyectar energía hacia el PCC, esta restricción está indicado en la ecuación (7), que para este caso es de $V_{d c_{\_} \text {minimo }}=639 \mathrm{~V}$.

$$
V_{d c_{\_} \text {minimo }}=\frac{2 \sqrt{2 V}_{f-n(r m s)}}{D_{q}}
$$

Si el nivel de tensión del banco de supercondensadores se encuentra por debajo de la mínima tensión establecida anteriormente, la referencia de corriente obtendrá un valor 0 para que de este modo no inyecte ni absorba energía durante la presencia de un hueco de tensión hasta que el nivel de tensión en el PCC sea superior a 238,95 V (establecido en la sección de este documento denominado "Calidad de Energía"). 


\section{DESEMPEÑO DEL SISTEMA DE CONTROL}

Para comprobar el desempeño del sistema basado en supercondensadores se considera los límites establecidos en la sección de este documento denominado "Calidad de Energía", a partir de ello se procede a simular el modelo de la Fig. 3 sin incluir el sistema de inyección de energía, el resultado del nivel de tensión está indicado en la Fig. 9.

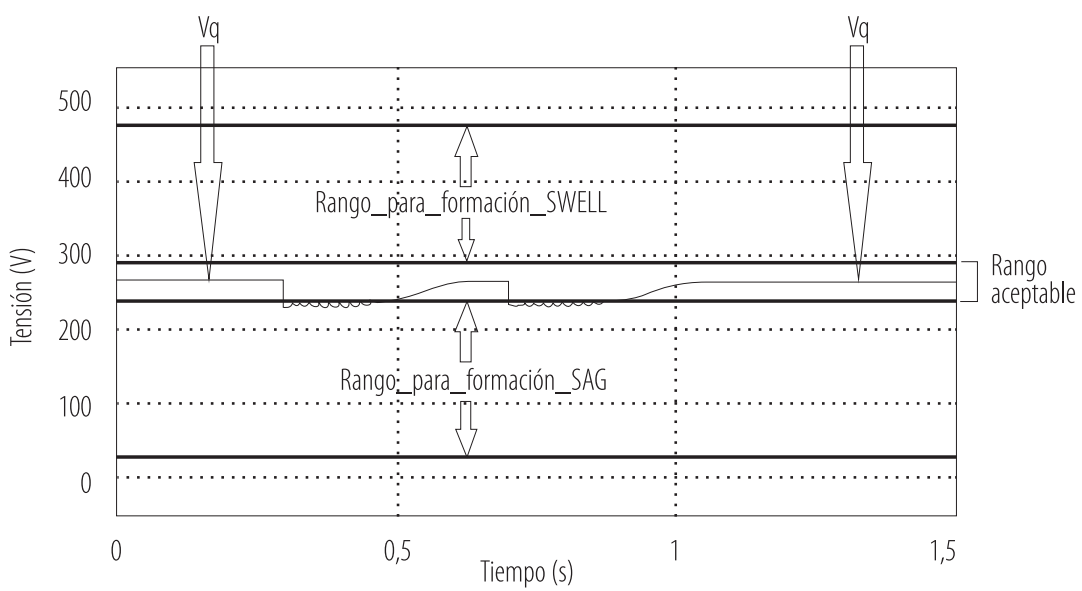

FIGURA 9. Comportamiento de tensión en PCC al momento del arranque de los motores en la red de distribución sin el sistema de energía basado en banco de supercondensadores

Al observar la Fig. 9, antes de ingresar en funcionamiento las cargas ( $t<0,3$ s), el nivel de tensión se encuentra en el rango aceptable (definido en la sección de "Calidad de energía) con un valor igual a 265,5 V y con una corriente de 13 A debido a la carga de $20 \Omega$ que se encuentra conectado y en 0,3 s se observa una disminución repentina y transitoria de la tensión con un valor igual a $231 \mathrm{~V}$, esto se debe a que en dicho momento arranca el primer motor produciéndose un pico de corriente de $400 \mathrm{~A}$, con un tiempo de duración de 0,2 s, producto por el cual la tensión en el PCC se ubica dentro del rango establecido para la formación de sags, el tiempo en que ingresa nuevamente el nivel de tensión dentro del rango aceptable es de 0,2 s, de igual modo sucede en 0,7 s debido a que ingresa a funcionar el segundo motor, con el mismo valor de pico de corriente y tiempo de estabilización.

Ahora se considera la conexión del sistema de energía basado en banco de supercondensadores, el cual inyecta energía para mitigar los huecos de tensión en el PCC obteniendo la Fig. 10. 


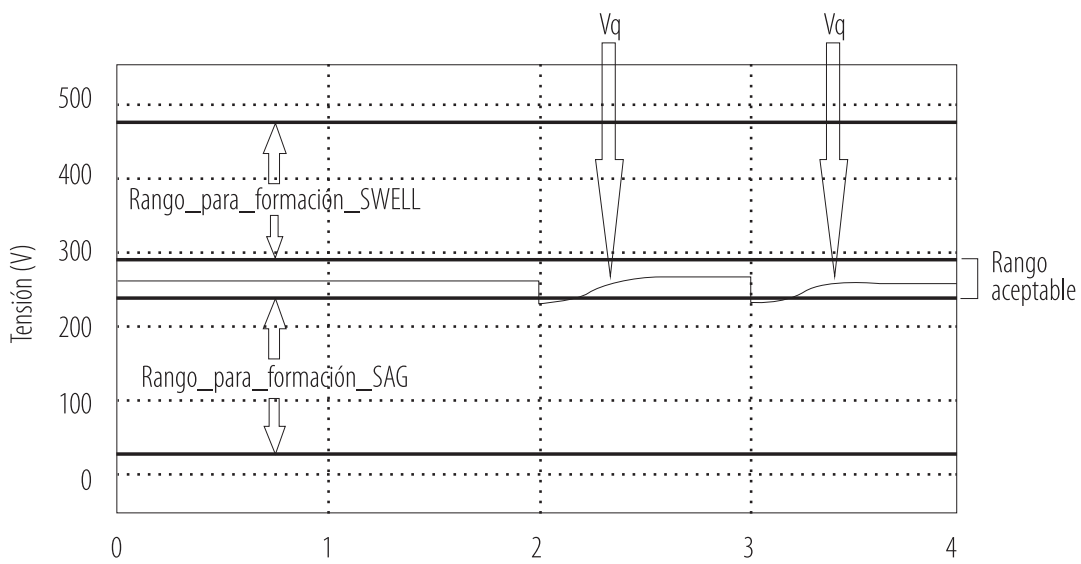

FIGURA 10. Nivel de tensión con la incursión del sistema de energía basado en banco de supercondensadores para mitigar los huecos de tensión en el PCC

En la Fig. 10, se puede observar que al momento del arranque del primer motor que ocurre en el tiempo de $2 \mathrm{~s}$ el nivel de tensión se encuentra dentro de los rangos aceptables, con un valor mínimo de $244 \mathrm{~V}$, en comparación con el nivel de tensión de la figura 9 se incrementó $13 \mathrm{~V}$, de igual modo sucede en $3 \mathrm{~s}$ cuando ingresa a funcionar el segundo motor con un nivel de tensión al momento del arranque de $244 \mathrm{~V}$.

El nivel de tensión del banco de supercondensadores se indica en la Fig. 11.

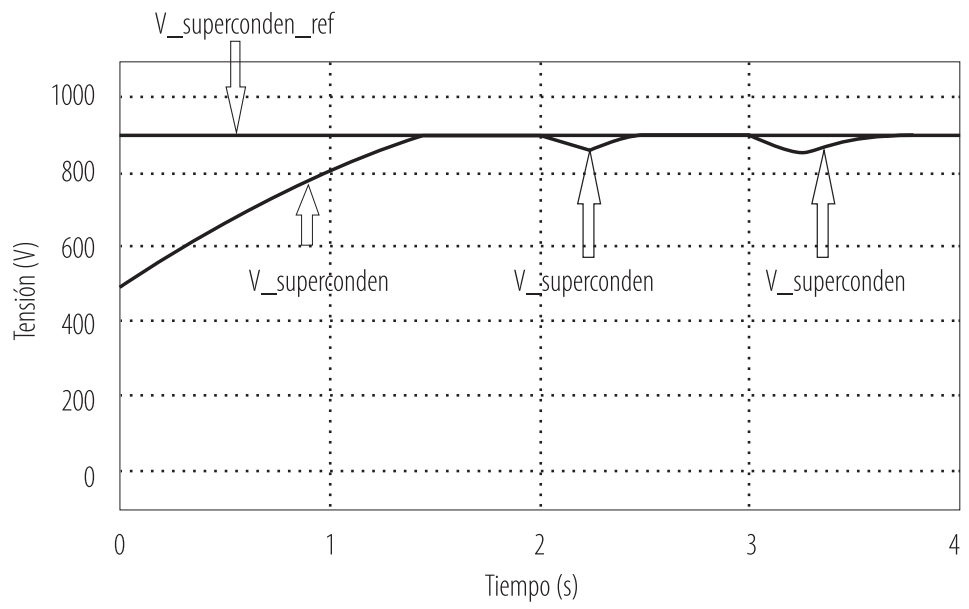

FIGURA 11. Nivel de tensión del banco de supercondensadores 
En la Fig. 11, se puede apreciar el nivel de tensión del banco de supercondensadores: antes de ingresar los motores a la red de distribución el nivel de tensión del banco de supercondensadores se incrementa hasta un valor de referencia de $900 \mathrm{~V}$, con el convertidor de potencia funcionando como rectificador, absorbiendo energía desde la red de distribución con referencia negativa igual a -50 A, cabe destacar que en esta condición la corriente del sistema de inyección basado en supercondensadores está desfasada con la corriente que circula a la carga (Fig. 12a), en este estado el sistema de inyección de energía basado en banco de supercondensadores se encuentra en modo carga; cuando la tensión del banco de supercondensadores alcanza su respectiva referencia consume una corriente igual a 2 A por fase, en este estado el sistema de inyección de energía se encuentra en modo stand-by; pero al momento del ingreso del primer motor existe una disminución de tensión del banco de supercondensadores con un valor mínimo de $858 \mathrm{~V}$ con su respectivo convertidor de potencia funcionando como inversor para que de esta manera suministre energía hacia la red de distribución, con su lazo de corriente con referencia positiva igual a $115 \mathrm{~A}$, de igual manera cabe destacar que la corriente del sistema de inyección basado en supercondensadores inyectada hacia la red de distribución se encuentra en fase con la corriente que circula hacia la carga (Fig. 12b), esta disminución de tensión del banco de supercondensadores se mantiene durante el arranque de los motores por un tiempo de 0,2 s, en este estado el sistema de inyección de energía se encuentra en modo descarga, una vez transcurrido ese tiempo la tensión del banco de supercondensadores se vuelve a incrementar hasta su valor de referencia (Fig. 11) repitiéndose nuevamente el ciclo mencionado anteriormente cuando exista la presencia de otro hueco de tensión.
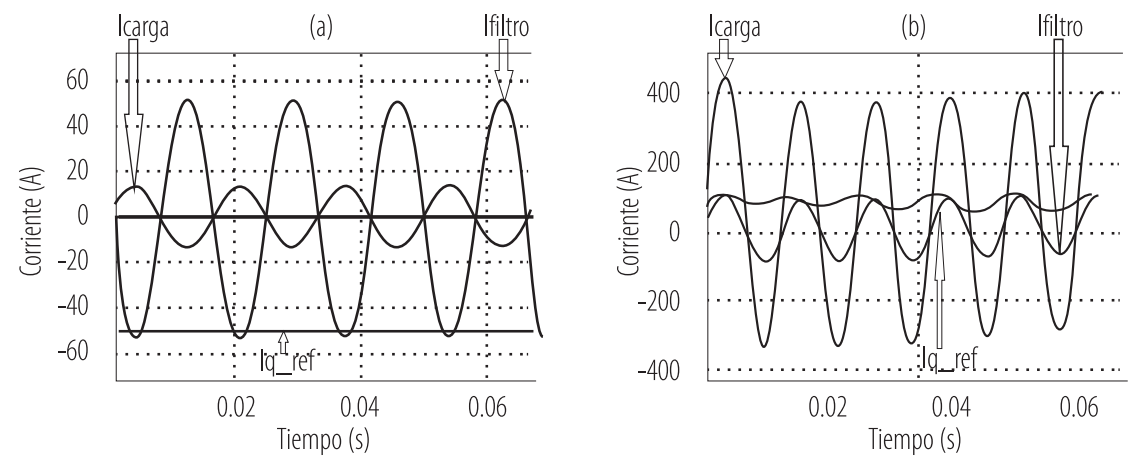

FIGURA 12. Corriente de referencia del sistema de energía basado en supercondensadores: a) en modo carga, b) en modo descarga

Un aspecto importante es el nivel de THD que se produce al momento de carga, standby y descarga del banco de supercondensadores que al simular se obtuvo lo siguiente: con respecto al THD de la corriente que circula hacia la carga se obtuvo 2,6\% menor a lo establecido que es del 8\% (regulación CONELEC 004/01, NP EN 50160-2010), con respecto al THD de la tensión medida en el PCC se obtuvo 2,3\% menor a lo establecido y finalmente el THD de la corriente que circula hacia el banco de supercondensadores con un valor de 4,2\% menor a lo establecido. 


\section{CONCLUSIONES}

El presente artículo realiza un estudio para mitigar los huecos de tensión en el PCC debido al ingreso de cargas transitorias a la red eléctrica, concluyendo así que el sistema basado en supercondensadores actúa de forma correcta ante una disminución de tensión en el PCC, pues dicho sistema posee una rápida respuesta de actuación evitando la formación de los huecos de tensión, otro aspecto que se concluye es que el nivel de tensión en el PCC no se ve afectado cuando el sistema de energía basado en banco de supercondensadores se encuentra en modo carga, finalmente se concluye también que el sistema de energía basado en banco de supercondensadores no afecta los niveles de THD de la tensión y corriente en el PCC.

Un problema que posee este sistema es que si el nivel de tensión de los bancos de supercondensadores se ubica por debajo de la tensión mínima permitida, el sistema ya no va a poder inyectar energía para evitar la formación de los huecos de tensión y si el sistema de control en ese instante actúa de forma errónea el sistema de inyección de energía va a empezar a absorber energía desde la red para ingresar al modo de carga involucrando de forma directa una disminución más drástica del nivel de tensión en el PCC. Para solucionar este inconveniente se debe instalar en paralelo otro sistema de inyección de energía auxiliar, el cual debe constar de una sistema de control que monitoree el nivel de tensión en los bancos de supercondensadores y en la red de distribución, pues de este modo cuando el nivel de tensión tanto de los bancos de supercondensadores y la red de distribución se ubique por debajo de la tensión permitida empiece a inyectar energía para evitar lo formación de los huecos de tensión en el PCC.

Este tipo de sistema de inyección de energía tiene la ventaja de que utiliza un solo convertidor de potencia trifásico involucrando un sistema de control no complejo que en comparación con [11] utiliza dos convertidores trifásicos controlados, pues el sistema de inyección de energía está basado en Flywheel de alta velocidad, lo que involucra un sistema de control más complejo, pues debe controlar al mismo tiempo la velocidad del motor síncrono de imanes permanentes (PMSM) y la tensión del Vdc-link.

\section{AGRADECIMIENTOS}

El primer autor agradece a la Secretaría de Educación Superior, Ciencia, Tecnología e Innovación (SENESCYT) de la República del Ecuador por la beca de cuarto nivel. El segundo autor desea agradecer el apoyo económico e institucional del Proyecto Prometeo patrocinado por la Secretaría de Educación Superior, Ciencia, Tecnología e Innovación (SENESCYT) de la República del Ecuador.

\section{CONTRIBUCIONES DE LOS AUTORES}

Carlos Orellana concibió la investigación, desarrolló la programación, producción de tablas y figuras y realizó el manuscrito; Luis González realizó la tutoría y revisión crítica del contenido intelectual del manuscrito; Filipe Tadeu y Nuno Abreu realizaron la tutoría. 


\section{REFERENCIAS}

[1] Bose A., Tomsovic K. \& Vaziri M. (2004). A Directed Graph Formulation of the Multistage Distribution Expansion Problem. IEEE Transactions on Power Delivery, 19, 1335 - 1341. doi: http://doi.org/10.1109/TPWRD.2004.829146

[2] TECSUP (2017). Programa de Extensión para profesional-Sistemas de Distribución de Energía Eléctrica. Recuperado de: https://es.scribd.com/document/179746191/sistemas-de-distribucion

[3] Banco Central del Ecuador (2017). Reporte de Minería. Dirección Nacional de Síntesis Macroeconómico. Recuperado el 11 de noviembre de 2017 de: https://web.archive.org/web/20170926215322/https://contenido.bce.fin.ec/ documentos/Estadisticas/Hidrocarburos/ReporteMiner0012017.pdf

[4] Información de Historia, Cultura y Turismo de Cuenca-Azuay (2017, mayo 06). Revista Cuenca llustre - Ecuador. Recuperado de: https://web.archive.org/web/20170616014309/https://patomiller.wordpress.com/

[5] Einzmann H. (1991). Tres Chorreras: Minería Artesanal e Informal en el Cantón Pucará. Ecuador: Adoum ediciones.

[6] Criollo H. \& Hernández F. (2017). Minería Artesanal del oro de Aluvión Mocoa, Putumayo, Amazonia Colombiana. Universidad Nacional de Colombia: Sede Amazonia-Leticia. Recuperado de: https://web.archive.org/ web/20170926220714/http://www.bdigital.unal.edu.co/53064/6/9587015630.preliminares.pdf

[7] Deshpande G. \& Sankeshwari S. (2013). Speed Control of Induction Motors Using Hybrid PI Plus Fuzzy Controller. International Journal of Advances in Engineering \& Technology, 2254 - 2258.

[8] González L. (2011). Mejora de la eficiencia y de las prestaciones dinámicas en procesadores electrónicos de potencia para pequeños aerogeneradores sincrónicos operando en régimen de velocidad variable (Tesis de doctorado). Universidad Politécnica de Valencia, España.

[9] Bardemaker F. (2006). Modulação Vetorial aplicada a Retificadores Trifásicos PWM Unidirecionais (Tese de doutorado). Universidad Federal de Santa Catarina, Brasil

[10] Barbi I., Morais A. \& Tofoli F. (2015). Modelling, Digital Control and Implementation of a Three-Phase Four-Wire Power Converter Used as A Power Redistribution Device. IEEE Transactions on Industrial Informatics, 12, 2 -5. doi: http://doi.org/10.1109/TII.2016.2544248

[11] Toliyat H., Talebi S., McMUllen P., Huynh C. \& Filatov A. (2005). Advanced High-Speed Flywheel Energy Storage Systems for Pulsed Power Applications. IEEE Electric Ship Technologies Symposium. doi: http://doi.org/10.1109/ ESTS.2005.1524703 